\title{
Offine/Online Optimum Routing of a UAV using Auxiliary Points
}

\author{
Abbas Fadavi $^{1}$, Ali Beheshti ${ }^{2}$, Pedram Kermani ${ }^{3}$ \\ ${ }^{1}$ Department of Mechatronics, Science and Research Branch, Islamic Azad Univercity, Semnan, Iran \\ ${ }^{2}$ School of Electracal Engineering, Iran University of Science and Technology, Tehran, Iran \\ ${ }^{3}$ Departmen of Electrical and Computer Eng, Abbaspour College of Engineering ShahidBeheshti University, AC., \\ Tehran, Iran
}

\begin{tabular}{|c|c|}
\hline Article Info & ABSTRACT \\
\hline Article history: & This paper presents a method to determine the route of a three-dimensional \\
\hline Received Jul 8, 2016 & $\begin{array}{l}\text { UAV. Three criteria; the height, the length of flight path and the un } \\
\text { authorized areas are used as the constraints and combined in a fuzzy function }\end{array}$ \\
\hline Revised Oct 21, 2016 & as the evaluation function. The article aimed to discover a minimum cost \\
\hline Accepted Nov 5, 2016 & $\begin{array}{l}\text { route from source to destination considering the constrains. In this paper a } \\
\text { new searching methodis proposed, with use of auxiliary points. The auxiliary }\end{array}$ \\
\hline Keyword: & $\begin{array}{l}\text { point method iterativelydivides a straight line to two shorter lines with less } \\
\text { cost of evaluation function. Implementation results show that the proposed }\end{array}$ \\
\hline Evaluation & $\begin{array}{l}\text { method dramatically decreasesthe calculations; meanwhile the ight route is } \\
\text { sub-optimum. }\end{array}$ \\
\hline
\end{tabular}

Copyright $\odot 2017$ Institute of Advanced Engineering and Science. All rights reserved.

Corresponding Author:

Abbas Fadavi,

Department of Mechatronics,

Science and Research Branch,

Islamic Azad Univercity, Semnan, Iran

Email: abbas_fadavi@yahoo.com

\section{INTRODUCTION}

UVA has its application mainly in the military industry [1-3]. However, it is used in a large number of non-civilian applications, such as fire fighting, meteorology, aerial photography and research projects. The aim of this article is to discover an appropriate route for the UAVs. An optimum route is a minimum distance route one which best satisfies the constraints. Different methods have been introduced and discussed in order to determine the optimal auxiliarypoints. Various articles have been presented to determine the optimal route forthe UVAs. A number of these articles have made use of classic methods. For example [4] and [5] use A for the purpose of searching, while [6] uses Teta and D. (A, Teta and D should be defined). Several articles have used evolutionary algorithms in order discover the route [7]. Article [8] took advantage of PSO, article [9] utilized GA and article [10] utilized GSO. Que et al. [11] applied hierarchical decision making. Their, the intended environment was first examined and divided into different areas. Then, according to the rate of the constraints satisfaction problems, the best identification areas and the paths were specified inorder to be able to reach the destination from the source among optimum zonesand to discover the best path. The method of Dijkstra was used. Robergeetal [12] presented a definite cost function. This function utilized a combination of different constraints like distance length with dangerous points and the rate of fuel consumption. Then, the optimal path was calculated using genetic algorithms or PSO based on minimizing or maximizing the cost function. Also, researchers like Kok et al [13] applied executive points to their hardware routing implementation. In article [11] the environment was first examined and divided into different zones. Then, optimal zones were identified. These are the zones which better satisfied the points inside the zones of 
constraints. Next, specific routes wereselected from the source to destination through optimal zones. To diagnose the best route, the method of Dijkstra was applied. Article [12] provided the certain performance function. It utilized a combination of various constraints such as dangerous spots along the route distance and fuel consumption amount; Followedby the discovery of the optimal routes by using this performance function withthe aid of PSO and GA. Article [13] explained the implementation of a routing method on the hardware. The second section presents definition of the problemand our mathematical approach to solving the problem. Then, their constraints are examined. The third section presents the proposed method and describes different methods useful in the discovery of the suitable auxiliary point. The fourth section presents the implementation results. The article is concluded in the end of the article.

\section{DEFINITION OF THE PROBLEM AND THE EVALUATION FUNCTION}

The problem of discovering the right way to move a UAV is an issue with a lot of input parameters and the answer is not unique. Therefore, this problem must be solved using mathematical modeling of the effective parameters to choose the path. In this problem, since it is impossible to pass the natural and synthetic obstacles, very low or very high altitude areas and unauthorized areas, some decisions are considered as constrains while other decision parameters are considered as an evaluation function. Practically, the problem of determining the optimal route becomes the mathematical maximizing or minimizing of the objective function with respect to the predefined constrains. The selected route involves the determination of the points (the length, width and height of each point), beginning from the point of origin and ending with the point of destination. In this paper, the speed of aircraft movement is constant and the path is determined was determined independently of speed. The intended provisions of the article contained height, length and unauthorized ight regions.

One of the solutions to cover all the defined constraints is the use of a phase machine to produce an overall indicator. In this method, all the information of the defined environment to cross and unauthorized areas to fly are given to the phasemachine. This phase car produces an indicator for each point of the environment which is called the desirable pass indicator. It covers all the defined constraints of the problem. The model obtained from the fuzzy machine is called a phase map. The aim is to obtain a route that better satisfies the desired pass indicator.

The modeling of [14] is adopted in this article in which the aforementioned three constraints have been modeled with the phase machine. The presented model specifies the performance function of the problem. This function is able to receive the position of any point in the environment and provide a certain amount as efficiency. A specification of the line between the starting and destinationpoint as the passing route was aimed at. This line consists of points. The position of points is placed in the performance function. The total of obtained amount of the performance function of points of a line shows the performance amount of the line. The route between the source and destination is optimalwhen the performance function is much less, which adheres better to the constraints.

To search the optimum points of the route different searching methods mightbe used. Two recently proposed methods, which we will compare our method with those, are the particle swarm optimization (PSO) is used in [8], and theGA [14]. This comparison is on the basis of the calculation time and better direction in regard to fuzzy map.

\section{THE OFFERED METHOD}

This section presents a method to discover an appropriate route between thesource and destination. Figure 1 shows a sample fuzzy map of an environment, inwhich each point of the map belongs to a fuzzy function. The constraints areinserted to a fuzzy function. Dark blue regions are preferred area with minimumcost and maximum performance and the red regions belong to non-authorized areas with maximum cost and minimum performance. In Figure 1 point $\mathrm{A}$ isconsidered as the source and point $\mathrm{B}$ as the destination. 


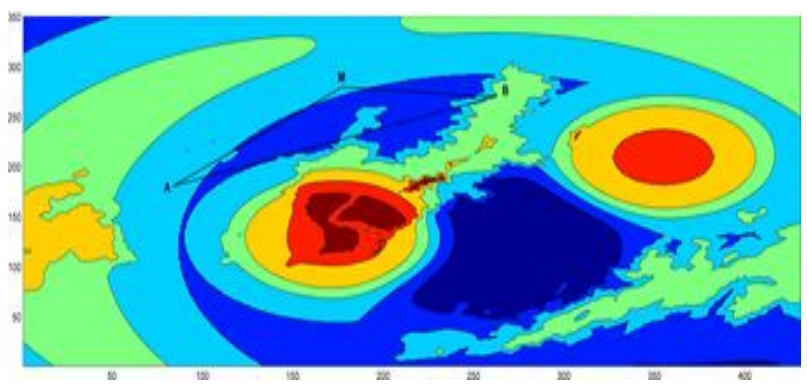

Figure 1. A hypothetical environment indicating statement of the problem A, Source B andauxiliary point M

\subsection{Decision-Making Regarding the Alternative Route}

It is a known fact that the closest path between the two points of $\mathrm{A}$ and $\mathrm{B}$ is the straight line $\mathrm{AB}$. Therefore line $\mathrm{AB}$ is the shortest path between $\mathrm{A}$ and $\mathrm{B}$. But as shown in the figure, line $\mathrm{AB}$ passes through an area with poor conditions. A path which is too short and does not pass through difficult areas is required. The point named $\mathrm{M}$ is considered as an auxiliary point. The line AM and MB are connected with the two points $\mathrm{A}$ and $\mathrm{B}$. This direction is longer than $\mathrm{AB}$, but does not pass through a difficult area.

If $\mathrm{F}$ is a cost function and $\mathrm{FAB}$ the cost of $\mathrm{AB}$ direction, If $\mathrm{FAB}>\mathrm{FAM}+\mathrm{FMB}$ Then the point $\mathrm{M}$ is a good point because it could help optimize the cost function. But if FAB $<$ FAM + FMB Then the point $M$ is not a good point because this could not optimize the cost function. The percentage of cost function improvement by the auxiliary point is called OPT and its relation is explained as follows:

$$
\frac{F_{A B}-F_{A M}-F_{M B}}{F_{A B}}=O P T
$$

In this relation, $\mathrm{A}, \mathrm{B}$ and $\mathrm{M}$ are source, destination and auxiliary point, respectively. If $\mathrm{OPT}>0, \mathrm{M}$ is the proper auxiliary point and if OPT $<0$, the auxiliary point does not act helpfully and only lengthens the route. If $\mathrm{M}$ is in the line $\mathrm{AB}, \mathrm{OPT}=0$. It should be noted that various constraints are available in the fitness function, and distance is just one of them. For this reason, in some cases, breaking a line into two lines by the auxiliary function increases the distance and decreases other constraints of the fitness function. Hence, the amount of the total fitness function generally decreases through the auxiliary point. The basic idea here was derived from the behavior of a human when encountering anobstacle. A human changes direction when facing an obstacle and tries to cross from one of its sides. Our proposed method is also the same is human decision making. OPT value indicates fewer costs, using the auxiliary point. If the valueis less than zero, it indicates that the same primary straight line between the two points is a good way and the use of auxiliary point does not improve the route.

By breaking a line and turning it into two lines, an auxiliary line will lead to a better route. In the same way, the Function F for the two obtained lines can be performed. By so doing, the straight line between the two points of source and destination is broken. Then, these two lines are converted into two other lines. This procedure is continued until the length of two lines becomes more than the minimum amount. Figure 2 indicates the result for the two hypothetical points. The route between the two points of A and B is divided into auxiliary points. In fact, these lines, with the help of a slight deviation in the route, enables for the satisfaction of the evaluation function. Classical artificial intelligence methods [15], solving the problems are categorized to bottom to top or top to bottom methods. In this research, our approach is top to bottom. This implies that the first issue was split into two parts, with each part further divided into smaller parts. Figure 3 demonstrates how this search occurs. Find Path function takes the points of source and destination, and finds the necessary auxiliary points.

First by using the function Find Acillary Point, the auxiliary point between the source and destination is determined. Then The OPT value is calculated. A lower value of OPT is lower than the minimum amount indicates that breaking the straight line between the source and destination is a sign of little profit. Therefore, the same straight line between the source and destination is announced as a desirable route. However, if the amount is more than the minimal amount, the direct line between the source and destination into two lines is broken with the help of auxiliary points. If the length of each of the two lines is more than the minimal amount, the algorithm can be repeated for each of the two broken lines. In fact, the straight line between the source and destination can be divided into such pieces that are very small, and the breaking of the beneficial parts becomes unnecessary. For example, in Figure 2, points A and B are source and 
destination, respectively. Point M1 is selected as an auxiliary point between these two points. A point between M1 and M4 is selected as the auxiliary point between points M1 and A. However, no point is selected between the points A and M4 because the straight line between the two points is suitable enough. It is possible to obtain an auxiliary point that reduces the computational cost. However, the improvement is so low that it can be ignored due to the computational cost. Between points M4 and M1, M2 auxiliary point is selected. The distance between the two points, M4 and M2, is small. For this reason, no auxiliary point has been selected between points M1 and M2.

Now the question is how to select the auxiliary point in the Find Auxiliary Point function. As it is clear from pseudo code, the destination-source position is specified as the input problem but the position of auxiliary points still remains unclear. The next section aims to answer the question of where is the optimal position for the auxiliary points.

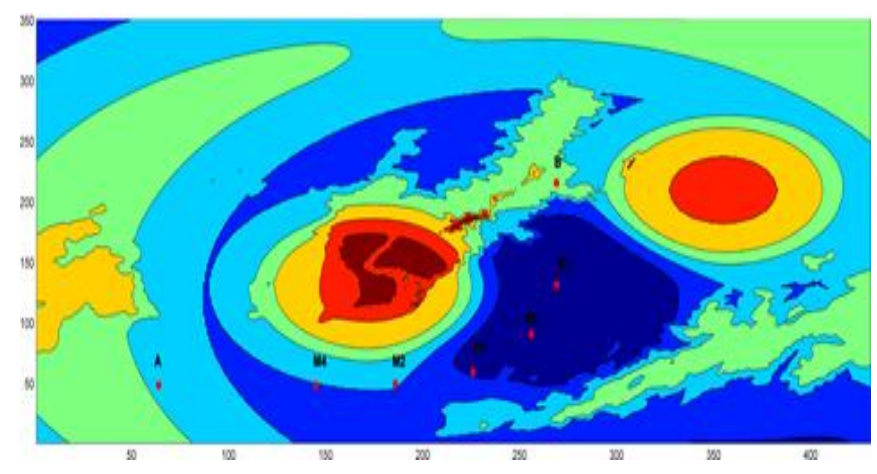

Figure 2. Displaying the auxiliary point between the two points of source and destination

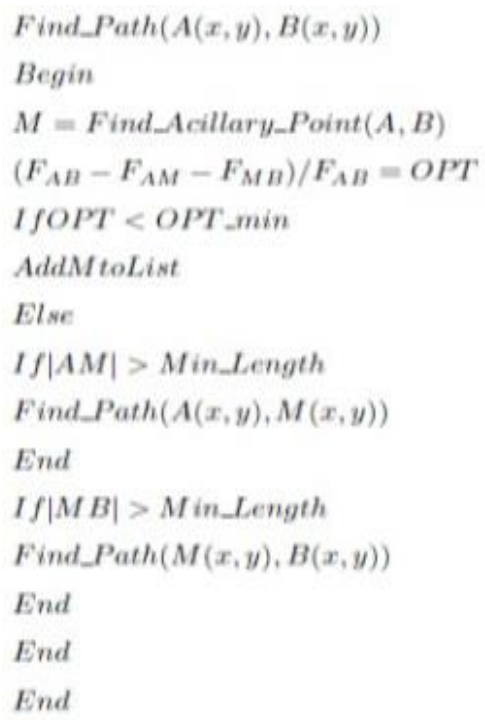

Figure 3. Find Path function takes the points of source and destination, and finds the necessary auxiliary points

\subsection{Finding a Suitable Auxiliary Point}

To search for the auxiliary point, there is need to limit the search space. Empirically, it was discovered that the optimal auxiliary point is located between thesource and destination. In Figure 4, point A is the source and B is the destination. In the first assumption, the square RSPQ were considered and the the auxiliarypoint was assumed in this rectangle. The length of RQ and SP is half of AB and is perpendicular to AB. As seen in the previous section, the discovery of the coordinates of the auxiliary point which can break the direct line into two new lines, and cause the largest decline in the value of the cost function was aimed at. 
This section tries to deal with how to search for the auxiliary point $M$ in the RSPQ rectangle. To do this, various methods are used.

\subsubsection{Full Search Method}

In the Full search method, all possible cases are examined. To find the auxiliary point, the points inside the rectangle are searched to find the best auxiliary point.

In this way, through examining all the points inside the rectangle and their OPT calculation, the point $\mathrm{M}$ is selected on the basis of maximized OPT. This will best help in the discovery of the auxiliary point, but the computational cost is very high.

\subsubsection{Using PSO}

To alleviate this problem, PSO [16] method can be used. This method consists of a set of particles, with each one looking for an optimal solution. Initially, each of the particles chooses an answer randomly. Then, by checking the answers and consulting the particles, they move to the better answer. Using every bit of its own experience and the best experience of all the particles, particles can move better.

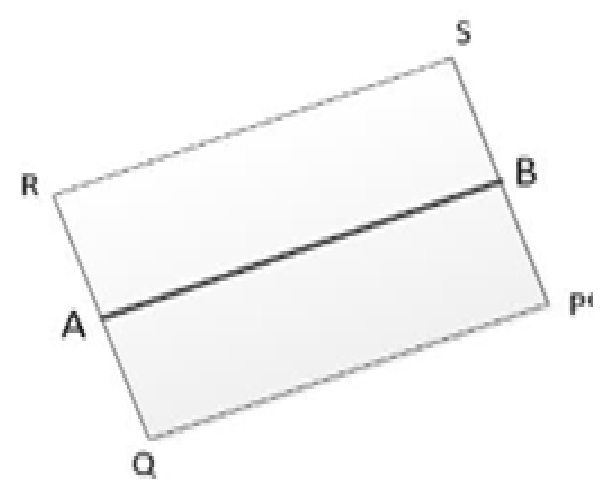

Figure 4. The points A and B are source and destination points. Search space rectangle limits the auxiliary points. The length of rectangle equals the distance of the source and destination and the width of the rectangle is half of it

The position of the suggested auxiliary point in the two-dimensional space is considered as the answer specifications of each particle and the points inside the RSPQ rectangle as the search space. A better position is one which better satisfies routing constraints. Some points become candidates in the search space randomly. Then, through PSO position, these points are optimized to be able to find the optimal auxiliary point. If an auxiliary point could not be found after several repetitions, the search will be stopped.

\subsection{The Use of Perpendicular}

Empirically, it was discovered that the optimal auxiliary point is mostly around or on the perpendicular line connecting the source and destination. Therefore, we assumed that the auxiliary point is on the perpendicular line of $\mathrm{AB}$. This assumption could only look on a perpendicular line to find an auxiliary point. The length of the perpendicular is limited to half of AB such as RS and PQ. By so doing, the checking points are greatly reduced.

\section{THE RESULTS OF SIMULATION}

In this section, the results of preforming the proposed algorithm are presented in a real environment. The considered environment in the problem is the real environment in Elburz Mountains. The dimension of the environment is 18,000 by 45,000, with the accuracy of 90 square meters. As presented in [14] the fuzzy map is a matrix of 200 by 500 . Each point in the matrix presenteda definite performance functions according to the three specified constraints.

The ofiered algorithm was implemented on MATLAB software. In each experiment, the source and destination were made clear by the user and the ofiered algorithm announced the test results as an output according to the performance function. 


\subsection{Determining the Route by Auxiliary Points}

Figure 5 and Figure 6 show, the routes of a UAV in two difierent environments and difierent fuzzy maps. The routes have been identified by auxiliary points according to the method described in 3.2. These auxiliary points were determined by the Full search method. Both figures related to fuzzy map are mentioned and have difierent routes.

As observed from Figure 5 and Figure 6, the route of movement was determined by the auxiliary point. This implies that the straight line between the source and destination is broken by auxiliary points.

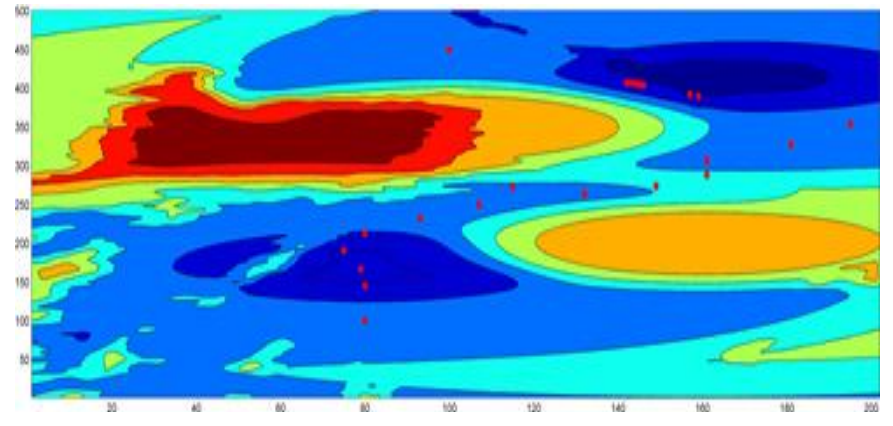

Figure 5. The auxiliary points determined by the Full search methodthe black points are source and destination and the red points the auxiliary points

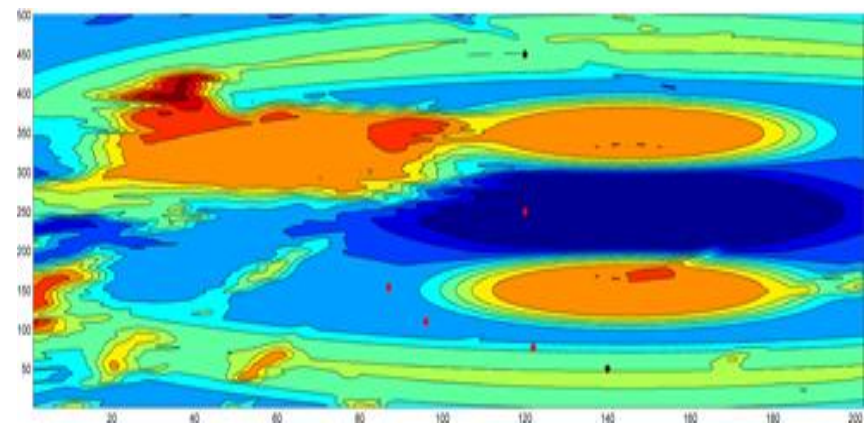

Figure 6. The auxiliary points determined by the Full search methodthe black points are source and destination and the red points the auxiliary points

To examine the optimization amount by auxiliary points, the following relation is expressed as the most complete one:

$$
\left(F_{A B}-F_{A M 1}-F_{M 1 M 2}-F_{M 2 M 3}-F_{M 3 M 4}-F_{M 4 M 5} \ldots \ldots F_{M n B}\right) / F_{A B}=O P T
$$

$\mathrm{n}$ is the number of segments into which the line $\mathrm{AB}$ is divided. For further examination, 100 pairs of source and destination points were considered randomly and the amount of average OPT is reported in the equation (1). This number indicated the positive effect of using the auxiliary points in determining the suitable route.

\subsection{Determining the Auxiliary Points by PSO}

The test for the same source, destination and environments of the previous part was repeated. The difference was the in the determination of the auxiliary point by PSO. The auxiliary point obtained from searching all the points was the best available auxiliary point. However, the auxiliary point obtained from PSO was one of the best available auxiliary points. For this reason, the obtained OPT of Full search was different from the OPT obtained from PSO.

The number of particles was set as 10 . The experiment was repeated 100 times. The mean value obtained by the auxiliary point with PSO was presented in table 1. As it is clear, this amount was less than the Full search OPT. 
One of the main factors in the search algorithm is the search time. In order to perform the algorithm online, the search time should be reduced as minimum as possible. In this article the average search time for finding the routes are presented for different searching methods. For better comparison average searching time of the the auxiliary point method with full search in the rectangle was considered as reference and other method are compared with this reference. Table 1 shows that search time with PSO in the same rectangle is only 0.06 of the full search method.

\subsection{Determining the Auxiliary Points on the Perpendicular}

The experiment was repeated for the same source and destination and the previous environments, with the exception that the auxiliary point on the perpendicular line connecting the source and destination was determined. OPT value obtained with the auxiliary points on the perpendicular line is shown in table 1 . As it is clear, this value was less than Full search OPT. Search time duration is shown in table 1.

\subsection{A Comparison of the Three Methods of Searching Auxiliary Points}

Three methods in an environment with the specified source and destination was performed, and the results shown with different colors in Figure 7. As it is clear from Figure 7, all three methods reached the answer, but each with different routes.

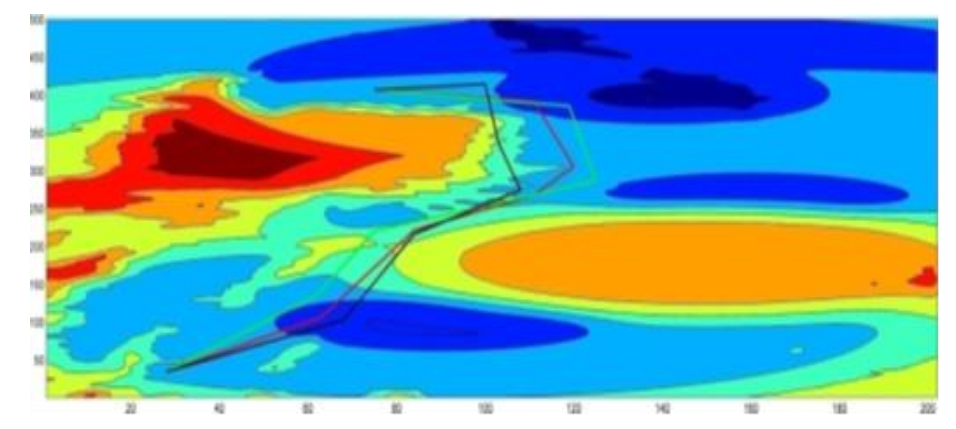

Figure 7. Routes running the Full search, PSO, and the perpendicular methods are shown by the colors red, green and black, respectively

\subsection{The Probability of the Optimal Auxiliary Point being Around the Perpendicular Line Connecting Source and Destination}

Figure 8 illustrates the ratio of the distance to the perpendicular line to the distance between the source and destination in terms of percentage. Each bar of the figure represents the ten percent total of the values. For example; the third bar represents the number of points whose percentage is between 20 and 29. As it is clear from Figure 8, the concentration of optimal auxiliary point is more around the perpendicular. The value of zero for the points means that they are on the perpendicular.In order to examine fewer points, the points on the perpendicular instead of thepoint around it are considered.

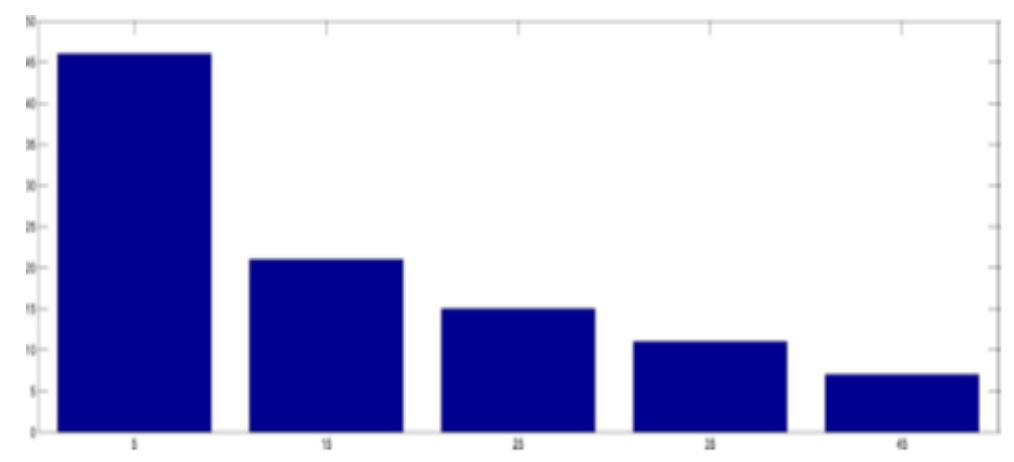

Figure 8. the ratio of the distance to the perpendicular line to the distance between the source and destination in percent 


\subsection{A Comparison with other Methods}

The proposed method was compared with the two methods of PSO [8] and GA [14]. For this reason all the three methods were run a hundred times in different environments and with different sources and destinations. In this paper, the offered method time was considered as the reference value it was compared with the times obtained from other methods.Tab.1shows the average of the obtained results. To implement PSO the same settings as specified in reference [8] were used. In some implementations PSO is caught in the local minimum and visually specified as failing to reach a satisfactory solution. Further implementation of PSO in this case does not have a good result. To alleviate the problem, the number of particles was increased. The problem was solved but the computational time increased. A reduction in the time calculations by reducing the number of particles would result in increasing the risk of falling into the local minimum. Consequently, the same number of reference particles [8] was used.

To implement the GA, similar specified settings in reference [14] was applied. It is noteworthy here in GA method, after completion of the minimum routing, the path was badly broken and comprised broken parts which can be softened by the softener specified in reference [14].

Table 1. Results of calculating the average performance and search time of different methods in hundred

\begin{tabular}{ccc}
\hline Method & OPT & Time \\
\hline PSO[8] & 10.9 & 2.3 \\
GA[14] & 12.1 & 0.7 \\
The use of auxiliary point using the Full search & 12.8 & 1 \\
The use of auxiliary point using PSO & 10.2 & 0.06 \\
The use of auxiliary point using the perpendicular & 8.3 & 0.023 \\
\hline
\end{tabular}

\subsection{The Examination of the Ratio of the OPT to the Route Visually}

In order to obtain a clear picture of the OPT, the following experiment was conducted. In this experiment, human intelligence was used to examine the quality of the route.A total of 25 routes were selected with the environment and source and destination, without having to consider how a route was created. The routes with the amount lower than two were not examined because they were noticeably false and contained aws. These directions were presented to three skilled users who were required to vote for any of the routes. Votes were either Terrible (0), Bad (1), moderate (2), good (3) Very Good (4). After voting, the average scores were obtained and compared with the average OPT routes. Figure 9 shows the ratio between the OPT and voted privileges. Because it is rare for the OPT route to be higher than 14, they were avoided in the graph. As it is clear from Figure 9, the routes with OPTs lower than 4 were specified as terrible and bad, while those higher than 8 were good or very good. Another issue indicated that the higher the OPT from the specified values; the more the route quality was believed to be saturated according to the viewers. Figure 7 confirmed this issue. Three paths have used three different methods but no much difference exists between their observed qualities.

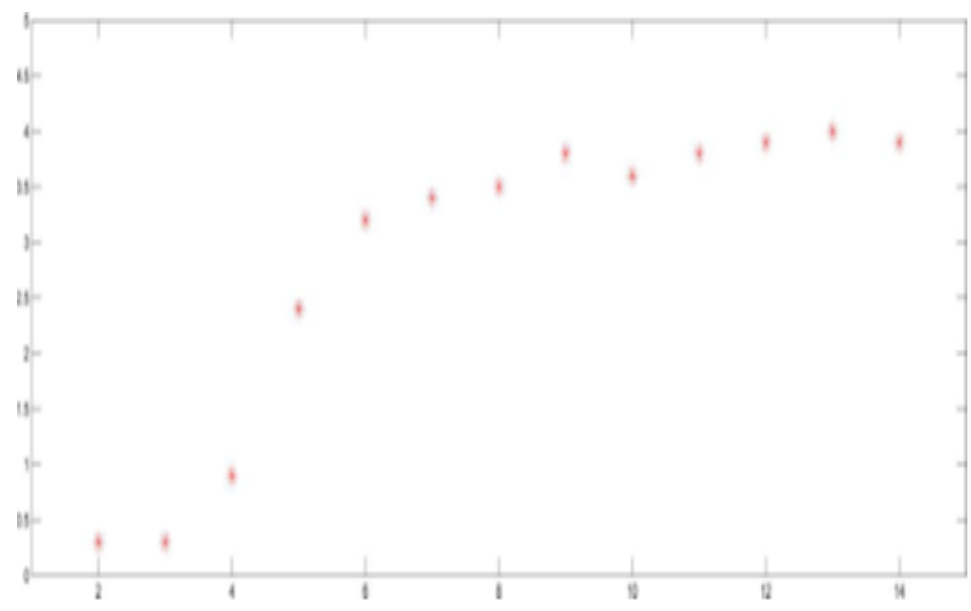

Figure 9. The ratio of the distance to the perpendicular line to the distance between the source and destination in percent 


\section{CONCLUSION}

In this paper a certain method was introduced to find a route for UAV based on searching auxiliary points in a rectangle with aspect ratio of 2:1. Moreover we showed that selecting the auxiliary point on the perpendicular dramatically decreases the computational complexity while the route is still desirable. The two parameters of OPT and speed were used to compare different methods with each other. It was found that the amount of OPT for the PSO search of auxiliary point in the rectangle proposed method possessed the best result compared with the other methods. The proposed method using the perpendicular possessed the best speed with desirable OPT. In fact, in the latter method a little accuracy is sacrificed for a lot of speed acceleration. The authors believe that if the calculations are going to be performed offline then finding the auxiliary point with full search in the rectangle is better. However, the search on the perpendicular is better for online calculations.

\section{REFERENCES}

[1] S.A. Gautam, N. Verma, "Path planning for unmanned aerial vehicle based on genetic algorithm \& artificial neural network in 3D", 2014 International Conference on Data Mining and Intelligent Computing (ICDMIC), pp. 1-5, 2014.

[2] Gao Yanhui and Xiao Qiangui and Hu Shousong and Ju Xiao, "Flight Control System Simulation Platform for UAV Based on Integrating Simulink With Stateflow", TELKOMNIKA Indonesian Journal of Electrical Engineering, vol. 10, pp. 985-991, 2012.

[3] Ali Moltajaei Farid, "UAV Controller Based on Adaptive NeuroFuzzy Inference System and PID", IAES International Journal of Robotics and Automation (IJRA), vol. 2, pp. 73-82, 2013.

[4] S.S. Jan, Y.H. Lin, "Integrated Flight Path Planning System and Flight Control System for Unmanned Helicopters", Sensors, vol. 11, pp. 7502-7529, 2011.

[5] P.P. Wu, D. Campbell, T. Merz,"Multi-objective four-dimensional vehicle motion planning in large dynamic environments", Systems, Man, and Cybernetics, Part B: Cybernetics, IEEE Transactions on, vol. 41, pp. 621-634, 2011.

[6] L. De Filippis, G. Guglieri, F. Quagliotti,"Path planning strategies for UAVS in 3D environments", Journal of Intelligent \& Robotic Systems, vol. 65, pp. 247-264, 2012.

[7] I.K. Nikolos, K.P. Valavanis, N.C. Tsourveloudis, A.N. Kostaras, "Evolutionary algorithm based offline/online path planner for UAV navigation", IEEE Transactions on Systems, Man, and Cybernetics, Part B: Cybernetics, vol. 33, pp. 898-912, 2003.

[8] J.J. Liang, H. Song, B.Y. Qu, X.B.Mao, "Path planning based on dynamic multi-swarm particle swarm optimizer with crossover", Intelligent Computing Theories and Applications, pp. 159-166, 2012.

[9] Xiao-Ting, Ji and Hai-Bin, Xie and Li, Zhou and Sheng-De, Jia, "Flight path planning based on an improved genetic algorithm", 2013 Third International Conference on Intelligent System Design and Engineering Applications (ISDEA), pp. 775-778, 2013.

[10] Y. Changqing, W. Zhurong, "UAV path planning using GSO-DE algorithm", TENCON 2013-2013 IEEE Region 10 Conference (31194), pp. 1-4, 2013.

[11] Y. Qu, Y. Zhang, Y. Zhang, "Optimal flight path planning for UAVs in 3-D threat environment", 2014 International Conference on Unmanned Aircraft Systems (ICUAS), pp. 149-155, 2014.

[12] V. Roberge, M. Tarbouchi, G. Labonte, "Comparison of parallel genetic algorithm and particle swarm optimization for real-time UAV path planning", IEEE Transactions on Industrial Informatics, vol. 9, pp. 132-141, 2013.

[13] J. Kok, L.F. Gonzalez, N. Kelson, "FPGA implementation of an evolutionary algorithm for autonomous unmanned aerial vehicle on-board path planning", IEEE Transactions on Evolutionary Computation, vol. 17, pp. 272-281, 2013.

[14] P. Kermani, A. Afzalian, et al., "Flight path planning using GA and fuzzy logic considering communication constraints," 2014 7th International Symposium on Telecommunications (IST), pp. 6-11, 2014.

[15] S. Russell, P. Norvig, A. Intelligence, "A modern approach", Artificial Intelligence. Prentice-Hall, Egnlewood Cliffs, vol. 25, pp. 65-71, 1995.

[16] Y. Shi, "Particle swarm optimization," IEEE Connections, vol. 2, pp. 8-13, 2004. 


\section{BIOGRAPHIES OF AUTHORS}
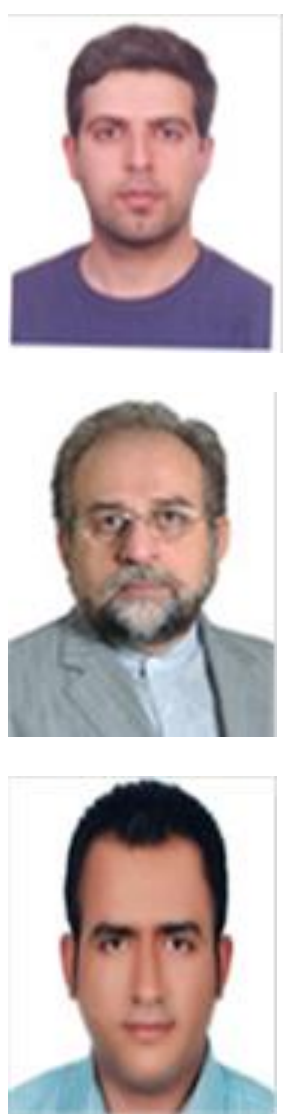

Abbasfadavi was born in Sari, Iran in 1978. He received the B.S. degree in electronic engineering from Azad university of Garmsar, Garmsar, Iran, in 2005 and the M.Sc. degree in Mechatronics from Science and Research Branch, Islamic Azad Univercity Semnan, Semnan, Iran in 2012. His research interests include Image Processing, Pattern Recognition, Algoritm Optimization, and Neural Networks.

Aliasghar Beheshti Shirazi He received his Ph.D.degrees in Digital Image Processing from Okayama University, Japan in 1995. Mr Beheshti was with IUST.

His research interests are in Image Processing and Codingand Signal Processing, Computer Networking, Data Communication system design, Satellite.

Emails Email: abeheshti (At) iust.ac.ir

Pedram Kermani received his B.Eng. degree in Electrical Engineering from Islamic Azad University, Tehran Iran, in 2008, and M.Eng. Degree in Control Engineering from Department of Electrical and Computer Engineering, Shahid Beheshti University, Tehran Iran, in 2012. He is currently a FPGA Desing Engineer at Recore Systems, Enschede Netherlands. His research interests autonomous aerial vehicles, path planning and artificial intelligence. 